\section{Medical Image Magnification Based on Original and Estimated Pixel Selection Models}

\author{
Akbarzadeh O. ${ }^{1,2,3 \odot *}$, Khosravi M. R..$^{4,5}$, Khosravi B. ${ }^{6}$, \\ Halvaee P. ${ }^{1}$
}

\begin{abstract}
Background: The issue of medial image resolution enhancement is one of the most important topics for medical imaging that helps improve the performance of many post-processing aspects like classification and segmentation towards medical diagnosis.

Objective: Our aim in this paper is to evaluate different types of pixel selection models in terms of pixel originality in medical image reconstruction problems. A previous investigation showed that selecting far original pixels has highly better performance than using near unoriginal/estimated pixels while magnifying some benchmarks in digital image processing.
\end{abstract}

Material and Methods: In our technical study, we apply two classical interpolators, cubic convolution (CC) and bi-linear (BL), in order to reconstruct medical images in spatial domain. In addition to the interpolators, we use some geometrical image transforms for creating the reconstruction models.

Results: The results clearly demonstrate that despite the absolute preference of the original pixel selection model in the first research, we cannot see this preference in medical dataset in which the results of BL interpolator for both tested models (original and estimated pixel selection models) are approximately the same as each other and for CC interpolator, we only see a relatively better preference for the original pixel selection model.

Conclusion: The current research reveals the fact that selection models are not a general factor in reconstruction problems, and the structure of the basic interpolators is also a main factor which affects the final results. In other words, some interpolators in medical dataset can be affected by the selection models, while, some cannot.

Citation: Akbarzadeh O, Khosravi MR, Khosravi B, Halvaee P. Medical Image Magnification Based on Original and Estimated Pixel Selection Models. J Biomed Phys Eng. 2020;10(3):357-366. doi: 10.31661/jbpe.v0i0.797.

\section{Keywords}

Image Interpolation; Image Reconstruction; Image Compression; Image Processing, Computer-Assisted; Image Enhancement; Benchmarking

\section{Introduction}

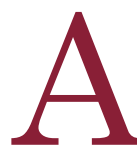
lthough spatial domain interpolation methods have been evaluated and compared, optimal performance evaluation for an interpolation algorithm is difficult to explain. Indeed, the suitability of a spatial domain interpolator is extremely associated with some specific factors considered. Furthermore, other items may also affect the
${ }^{1} \mathrm{MSc}$, Department of

Biomedical Physics and

Engineering, Shiraz

University of Medical Sci-

ences, Shiraz, Iran

${ }^{2} \mathrm{MSc}$, Department of

Communications and

Electronic Engineering,

Shiraz University, Shiraz,

Iran

${ }^{3} \mathrm{MSc}$, Department of

Electronics and Telecom-

munications, Politecnico

di Torino, Italy

${ }^{4} \mathrm{PhD}$, Department of

Electrical and Electronic

Engineering, Shiraz

University of Technology,

Shiraz, Iran

${ }^{5} \mathrm{PhD}$, Department of

Computer Engineering,

Persian Gulf University,

Iran

${ }^{6} \mathrm{MSc}$, Department of

Material Science and

Engineering, Sharif

University of Technology,

Tehran, Iran

*Corresponding author:

O. Akbarzadeh

Department of Biomedi-

cal Physics and Engi-

neering, Shiraz Univer-

sity of Medical Sciences,

Shiraz, Iran, Department

of Communications and

Electronic Engineering,

Shiraz University, Shiraz, Iran

E-mail: omidakbarza-

deh82@gmail.com

Received: 18 June 2017

Accepted: 10 August 2017 
choice of an interpolation method and the precision of its outputs. The definition of modelling is to create a series of frameworks in order to imitate a real system. A model will not be the same as the reality, and the model which imitates the reality. In many barriers, the processes performed are based on a model. In this article, the aim is to analyze modelling performed for the special problem of interpolationof medical images based on the directly original and indirectly estimated pixel selection models. Interpolation based on spatial estimation can be used for various purposes such as image de-noising and restoration [1], image zooming/magnification and reconstruction [1], image security [2], image fusion [3-4], image compression [5] and image de-mosaicking [56].Therefore, image interpolation approaches play a pivotal role in medical image processing. They are wanted for many feasible applications of medical imageprocessing such as computed tomography (CT), and magneticresonance imaging (MRI). In these images, the reconstruction could utilize interpolation. In new X-ray imagingsystems such as digital subtraction angiography (DSA),interpolation provides assistance to enable the computerboosted regulation of the current radiograph and the mask image. In addition, interpolation for focusing or revolving medical images after their acquirement is usually utilized in diagnosis and cure, and interpolators are combined into systems for computer-aided diagnosis (CAD), computer-assisted surgery (CAS) and image transmission, communication systems [7-8] and surveillance systems [9]. For example, if under interpolation, the total size of an image is reduced to1/4 of its first size, it means that each dimension of the image has been reduced to a half size.

As follows, we firstly review the basic concepts of image resizing problems and digital resolution in digital images. In the third section, we introduce our reconstruction models based on two different pixel selection approaches. In fourth and fifth sections, evalu- ation scheme and numerical results are investigated. The final section is allocated to the conclusion.

\section{Material and Methods}

\section{Image Fundamentals}

This study is a technical research with focus on simulation and computer-assisted image modeling to investigate image reconstruction modeling.

The digitization of images can often constitute two simultaneous processes of sampling and quantization. These two procedures are briefly described in order for the consideration of image storage requirements. Sampling process is used to digitize the spatial information in an image. It is often reached by dividing an image into a square, rectangular and newly hexagonal array of sampling points. Each of the sampling spots is referred to as a photo elementcalled a pixel.Ademonstration of a digital image is provided when consisting of a central dark area with the light climbing towards the periphery, see Figure 1. The process may be summarized as the digitization of an analog image into an $\mathrm{N} \times \mathrm{N}$ array of pixels. A sample value for $\mathrm{N}$ is 128 for a nuclear medicine scan, 512 for CT and MRI scans, 1024 for a DSA image and 2048 for a computed radiography image and digital radiograph. Note that $\mathrm{N}$ has

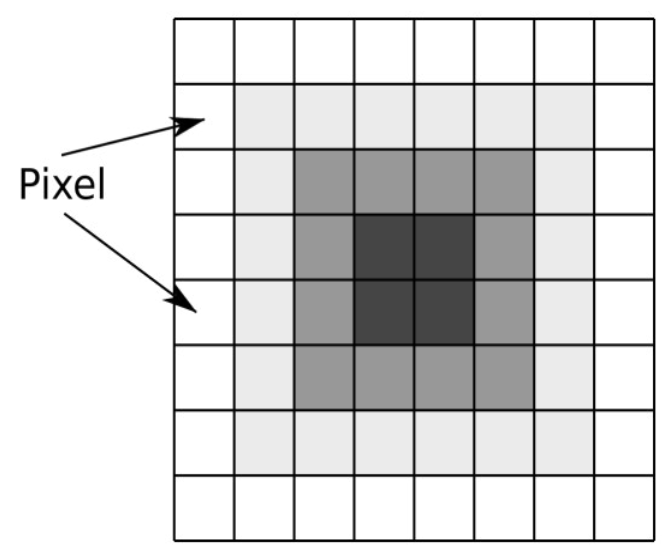

Figure 1: A representation of a digital image 
Medical Image Magnification

weights which are round number and powers of 2 , because of the binary nature of modern computing sort.Naturally, the bigger number of pixelsdenotes higher spatial resolution of the digitized image.

The number of bits, $b$, is essential to show an image in digital format. It is explicit that the bit number is given by $\mathrm{b}=\mathrm{N} \times \mathrm{N} \times \mathrm{M}$ for a square array with $\mathrm{N}$-pixel and the radiometric accuracy of M-bit. The following chart shows that the number of bits is vital to represent images that are digitized. It must be known that very huge values are required to attain the resolution, which is utilized in medical imaging. Spatial accuracy is not the only factor in determining the spatial resolution of an image, because, on the other hand, the dot per inch (DPI) value is another effective factor in the spatial resolution of an image. In Figure 2, although the dimensions of both images HR and HR' are equal, HR' image has less DPI than image HR, so this fact reveals the resolution of an image does not depend on its sole size. If no methods of spatial estimation (interpolation) are used for resizing the image, then, $\min \left\{D P I_{H R}\right\}=1 / 4 D P I_{H R}$, but the use of appropriate estimator (interpolator) causes $1 / 4 D P I_{H R}<D P I_{H R^{\prime}}<D P I_{H R}$. In the example shown in Figure 2, the rate of compression of data is 0.75 , as Eq. (1), that is, $0.75 \%$ of the primary data has been omitted (we have assumed that any type of image coding has not been used except the resizing-based ap-
(HR)

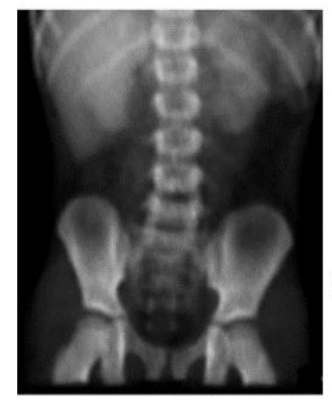

Original Image $(2 \mathrm{M} \times 2 \mathrm{~N})$
(LR)

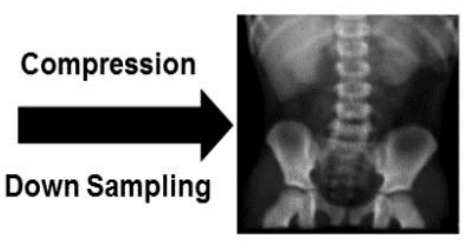

$(\mathrm{M} \times \mathrm{N})$

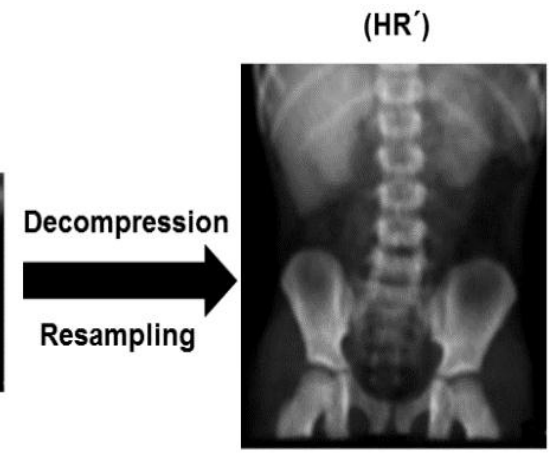

$(2 \mathrm{M} \times 2 \mathrm{~N})$

Figure 2: A workflow of compression process through the interpolation methods; HR, LR and $H R^{\prime}$ denote high resolution, low resolution and reconstructed images, respectively.
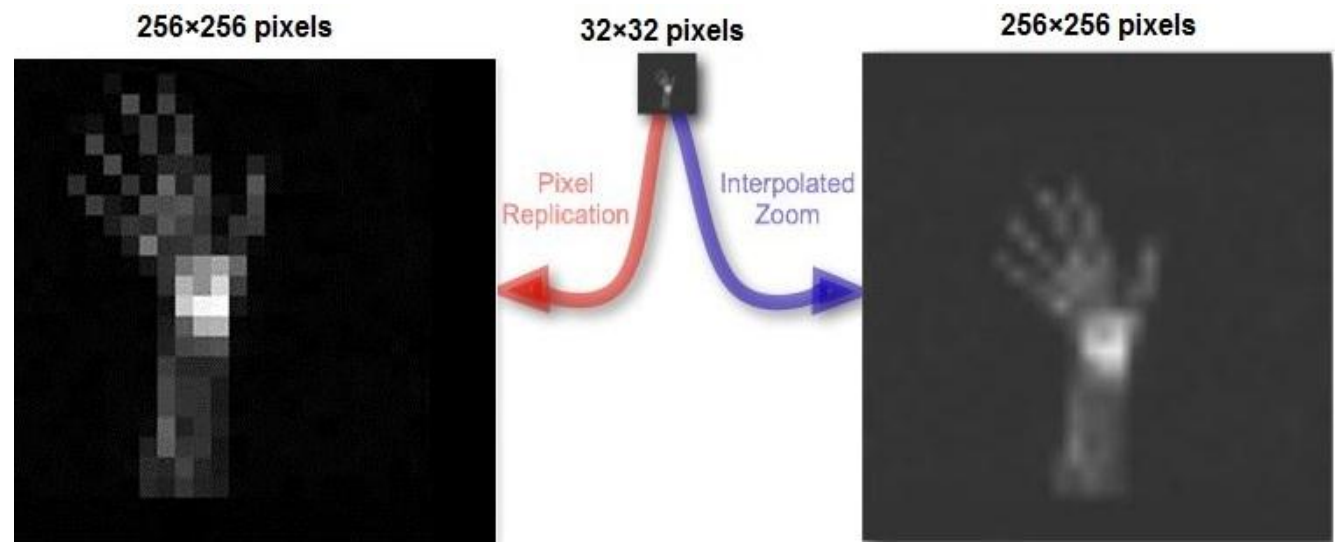

Figure 3: Images magnified based on repetition and interpolation of pixels. 
proach). Figure 3 shows the importance of image interpolation that prevents the blocking issue of the simple pixel replication (For more detail, see Table 1).

$$
\begin{gathered}
\text { Compression Rate (C.R. })=1-\left(b^{\prime} / b\right) \\
\text { If } b^{\prime}=\frac{1}{4} b \rightarrow \text { C.R. }=0.75
\end{gathered}
$$

For a primary HR image with 700dpi resolution (dpi is the unit of DPI factor) which has the dimension of $512 \times 512$ (pixels) and the depth of 8-bit, the primary volume is 216.32 (bits), which in the end, the total volume of HR' will be $216.32 / 4$ bits, but with a DPI it is less than $300 \mathrm{dpi}$.

\section{Image Reconstruction}

In compression of the information, changing compressed information into an uncompressed state is called decompression. To do so, we use the methods that are called reconstruction methods. There are various methods of reconstruction used; this way, re-sampling is one of the most useful tools. Re-sampling can be the sum of two operations up-sampling for resizing and estimating methods like spatial domain interpolators. In many aspects of image processing, the spatial domain estimation means the same as interpolation method. The most important geometrical transform in order to do resizing in digital images is an affine linear transform as Eq. (2), where the output is consequently obtained by Eq. (3). According to Eq. (3), $(X, Y)$ are the primary coordinates of pixels, $\left(X^{\prime}, Y^{\prime}\right)$ and are the secondary coordinates of the pixels in resized (up-sampled) image. It is clear that after resizing, there is a possibility of producing new pixels, which should be estimated by an estimation method; the estimation is done based on the existing values of adjacent pixels.

$$
\begin{aligned}
& \left(\begin{array}{l}
X_{H R^{\prime}} \\
Y_{H R^{\prime}} \\
Z_{H R^{\prime}}
\end{array}\right)=\left(\begin{array}{ccc}
c_{x} & 0 & 0 \\
0 & c_{y} & 0 \\
0 & 0 & 1
\end{array}\right)\left(\begin{array}{l}
X_{L R} \\
Y_{L R} \\
Z_{L R}
\end{array}\right) \\
& X_{H R^{\prime}}=c_{x} X_{L R} \\
& Y_{H R^{\prime}}=c_{y} Y_{L R}
\end{aligned}
$$

Figure 4 shows the way of resizing (upsampling) for the sample image with the size of $2 \times 2$ whose dimensions have been doubled in both of length and width $c_{x}=c_{y}=2$. Af-

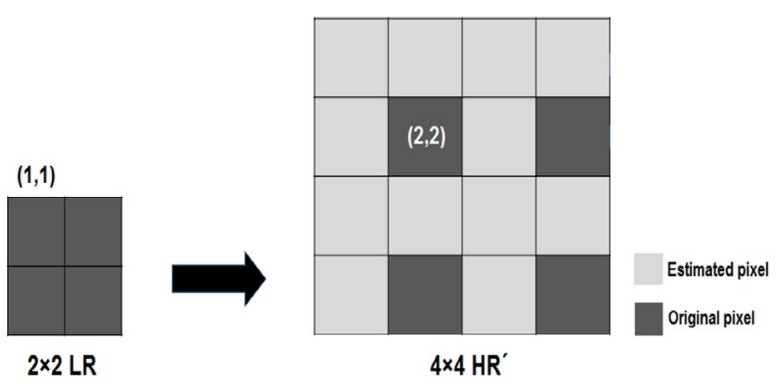

Figure 4: Up-sampling based on standard linear affine transform.

Table 1: The number of bits, which is essential to save non-coded images digitized to spatial resolution of $\mathrm{N} \times \mathrm{N}$ pixels and depth of $\mathrm{M}$.

\begin{tabular}{cccc} 
& \multicolumn{3}{c}{ Radiometric Accuracy (Depth) } \\
\cline { 2 - 4 } Spatial Accuracy $\mathbf{( N \times \mathbf { N } )}$ & $\mathbf{M = 1 0}$ & $\mathbf{M = 1 2}$ \\
\hline $128 \times 128$ & 131,072 & 163,840 & 196,608 \\
$256 \times 256$ & 524,288 & 655,360 & 768,432 \\
\hline $512 \times 512$ & $2,097,152$ & $2,621,440$ & $3,145,728$ \\
$1024 \times 1024$ & $8,388,608$ & $10,485,760$ & $12,582,512$ \\
\hline $2048 \times 2048$ & $33,554,432$ & $41,943,040$ & $50,331,648$
\end{tabular}


ter performing the required rescaling, the next step is to estimate values for the new pixels through interpolation methods. To specify a better estimation method, a technical assessment is required by numerical standards of quality assessment (QA) which is based on similarity evaluation of outputs, different interpolators and the source HR image. In the science of computer graphics, it can be shown that when similarity of a reconstructed image is more then another one to the source HR image, then, its DPI value will be greater than the second case equivalently [1]. There are various estimation methods based on interpolations such as classic methods, cubic convolution (BL) and bi-linear (CC) [1, 10-12] used in this study. We investigate pixel selection models under these two interpolation methods. More explanations of the interpolation methods can be found in [1].

\section{Experiment Design}

In order to investigate the effects of various estimators, we have to design a test. To this end, first, we have to change the primary image (HR) into LR version through down-sampling, then, through reconstruction, it is changed into HR' which has equal dimensions to the source HR image. In order to have a numerical quality assessment (QA), we can use some metrics such as PNSR [13] or SSIM [3] which obtain the order of similarity between HR and HR' images. PNSR is as Eq. (4) and is used here. Although it does not match human visual system (HVS) [4] well, it has been known as an acceptable standard metric, and is one of the most well-known standards of quality assessment in digital image processing (DIP).

$$
\begin{gathered}
M S E=\left(\frac{1}{M N} \sum_{i=1}^{M} \sum_{j=1}^{N}\left(f_{i j}-f_{i j}^{\prime}\right)^{2}\right) \\
R M S E=\sqrt{M S E}=\left(\frac{1}{M N} \sum_{i=1}^{M} \sum_{j=1}^{N}\left(f_{i j}-f_{i j}^{\prime}\right)^{2}\right)^{\frac{1}{2}} \\
P N S R=20 \log \frac{255}{R M S E} \quad(\text { for } 8-\text { bit images })
\end{gathered}
$$

The mapping used between two images under re-sampling and down-sampling processes can be clearly effective on outputs and some parts of modeling where error comes from these two stages; however, our objective here is not the error of this section. Thus, for all tests, an equal mapping must be used in the stage of re-sampling (a design like Figure 4) and down-sampling (a design like Figure 5). The design used in down-sampling stage is based on Eq. (5) or equivalently Figure 5 under the condition of $c_{x}=c_{y}=1 / 2$.

$$
\begin{gathered}
X_{L R}=\frac{X_{H R}+\left(c_{x}\right)^{-1}-1}{\left(c_{x}\right)^{-1}} \\
Y_{L R}=\frac{Y_{H R}+\left(c_{y}\right)^{-1}-1}{\left(c_{y}\right)^{-1}}
\end{gathered}
$$

\section{Results}

\section{Modeling Results}

Our goal is to do compression with the rate of C.R. $=1-(1 / 16)=0.94$, that is, we compress images with the dimensions of $512 \times 512$ up to $128 \times 128$. To do this, we will simply use Eq. (5) under $c_{x}=c_{y}=4$. The problem which should be discussed is the number of re-sampling steps. It is clear that it is possible to do the image reconstruction directly and produce the HR' image (or HR' direct). In this state, we use Eq. (3) under $c_{x}=c_{y}=4$, but another

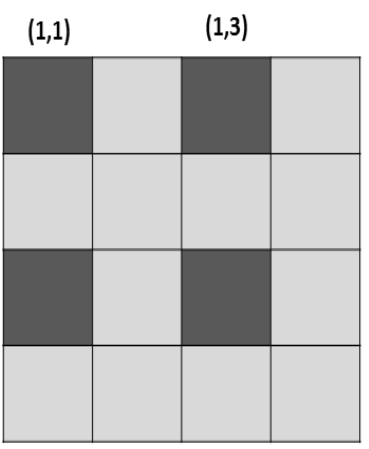

$4 \times 4 \mathrm{HR}$
$(1,1) \quad(1,2)$

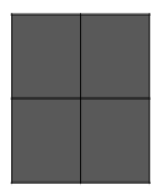

$2 \times 2$ LR
Figure 5: Down-sampling model used. 
way is to perform re-sampling in two steps, we again use Eq. (2) under $c_{x}=c_{y}=2$ in order to produce HR' (or HR' indirect), whose dimensions are $256 \times 256$ in the first step, and $512 \times 512$ in the second step. The reason of using multi-step state is that in many scientific articles dealing with interpolation methods, the number of steps is not usually investigated most of the time, this work is done in only one-step, whereas many new methods for spatial domain estimation are not capable to do the reconstruction in a step like LMMSE [3-4]. Thus, there is the potential to change such algorithms to the direct mode of estimation (i.e. one-step); if the direct approach in an application for a wide range of scalable (freesize) interpolators has better performance. For instance, LMMSE method is one, which will do the mentioned problem in two steps. Therefore, it is evident that, in a case of comparing this method with other methods, it is desired to change other methods such as LMMSE to produce another version, which is capable of estimating in just one-step (or directly). Basically, two interpolation methods should be compared when all conditions of modeling are the same as each other. It is therefore clear that the first step is the equality of the dataset, and the metrics of QA that are the general requirements of simulation; nonetheless, these two are not all the conditions, the implementation of models is also very important. In Table 2, the results of PNSR metric for test images including benchmarks such as Lena, Baboon, etc. are seen [14]; where all images have been tested in terms of four models based on original/direct and estimated/indirect pixel selection models and also two interpolators of $\mathrm{BL}$ and $\mathrm{CC}$ (they are therefore named Direct BL, Indirect $\mathrm{BL}$, Direct $\mathrm{CC}$, and Indirect $\mathrm{CC}$ ). In Table 3, PNSRs of all models for 8 medical test images (Figure 6) are shown.

The results clearly show that despite the absolute preference of original pixel selection model in the first research [14], we cannot see this preference in medical dataset in which
Table 2: PNSRs of all models for the benchmarks.

\begin{tabular}{ccccc}
$\begin{array}{c}\text { Sample } \\
\text { Image }\end{array}$ & $\begin{array}{c}\text { Direct } \\
\text { BL }\end{array}$ & $\begin{array}{c}\text { Direct } \\
\text { CC }\end{array}$ & $\begin{array}{c}\text { Indirect } \\
\text { BL }\end{array}$ & $\begin{array}{c}\text { Indirect } \\
\text { CC }\end{array}$ \\
\hline Baboon & 23.1811 & 23.5885 & 22.9007 & 23.5320 \\
\hline Barbara & 23.3498 & 23.6039 & 23.1909 & 23.5833 \\
\hline Boat & 25.0423 & 25.5370 & 24.7197 & 25.4792 \\
\hline Lena & 29.2312 & 30.0148 & 28.7892 & 29.9396 \\
\hline Peppers & 28.4763 & 29.2906 & 28.0100 & 29.2214 \\
\hline Plane & 26.3751 & 27.2243 & 25.8854 & 27.1464 \\
\hline Sailboat & 24.7903 & 25.5305 & 24.3578 & 25.4600 \\
\hline Tiffany & 26.1302 & 26.5471 & 25.8921 & 26.5110
\end{tabular}

Table 3: PNSRs of all models for medical test images.

\begin{tabular}{lcccc}
$\begin{array}{c}\text { Sample } \\
\text { Image }\end{array}$ & $\begin{array}{c}\text { Direct } \\
\text { BL }\end{array}$ & $\begin{array}{c}\text { Direct } \\
\text { CC }\end{array}$ & $\begin{array}{c}\text { Indirect } \\
\text { BL }\end{array}$ & $\begin{array}{c}\text { Indirect } \\
\text { CC }\end{array}$ \\
\hline Image1 & 32.981 & 32.8670 & 32.2649 & 32.4277 \\
\hline Image2 & 32.651 & 32.8475 & 32.4645 & 32.7899 \\
\hline Image3 & 36.129 & 36.4860 & 35.5892 & 35.9414 \\
\hline Image4 & 34.087 & 35.6459 & 33.9790 & 34.0205 \\
\hline Image5 & 30.543 & 31.7586 & 30.0479 & 30.9286 \\
\hline Image6 & 35.451 & 36.2075 & 34.6418 & 35.9688 \\
\hline Image7 & 34.098 & 35.3335 & 34.0089 & 35.6231 \\
\hline Image8 & 33.283 & 33.8882 & 33.7415 & 34.0013
\end{tabular}

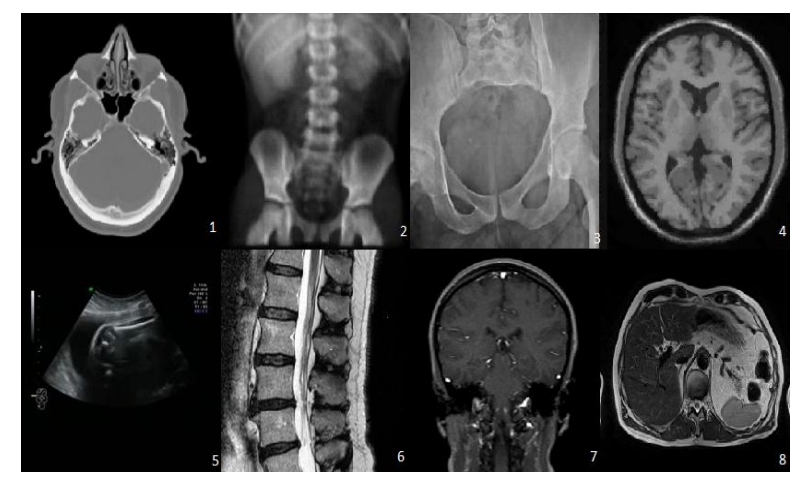

Figure 6: Medical test images contain various types of images, e.g. MRI, Ultrasound, $\mathrm{X}$-ray images. 
the results of BL interpolator for both tested models (original and estimated pixel selection models) are nearly the same as each other. For CC interpolator, we only see a relatively better preference for the original pixel selection model. Figures 7 and 8 also describe the data of Table 3 for BL and CC interpolators separately (under two different pixel selection models).

Eq. (6) is a simple metric for computing the distance or difference between two selected models. The results of using this metric are shown in Figure 9, and Table 4 describes its details. It is clear that "d13" has the least value among all labels, because Direct BL and Indirect BL models in medical dataset nearly have the same PSNRs.

$$
d_{i j}=\sqrt{\sum_{\text {all images }}\left(P N S R_{i}-P N S R_{j}\right)^{2}}
$$

Figure 10 shows some processed images under the mentioned models visually. See more details in its capture. Visual comparison has usually been a main factor for quality assessment.

\section{Discussion}

This paper gives a complementary viewpoint on findings in [14]. The results clearly demonstrate that despite the absolute preference of the original pixel selection model in the first research, we cannot see this preference in medical dataset in which the results of BL interpolator for both tested models (original and estimated pixel selection models) are approximately the same as each other and for $\mathrm{CC}$ interpolator, we only see a relatively better preference for the original pixel selection model. The current research reveals the fact that selection models are not a general factor in reconstruction problems, and the structure of the basic interpolators is also a main factor which affects the final results. Therefore, in designing new magnification algorithms for direct zoom of medical images, it is possible

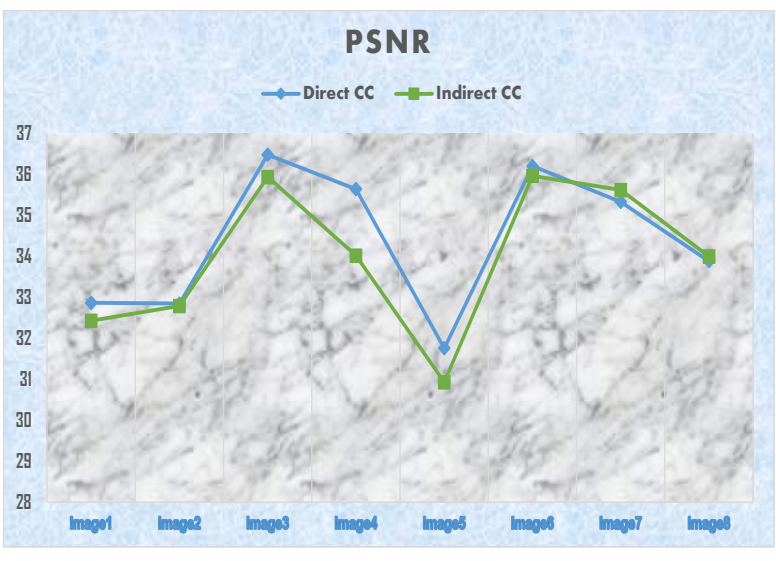

Figure 7: CC-based results for direct and indirect models in medical images.

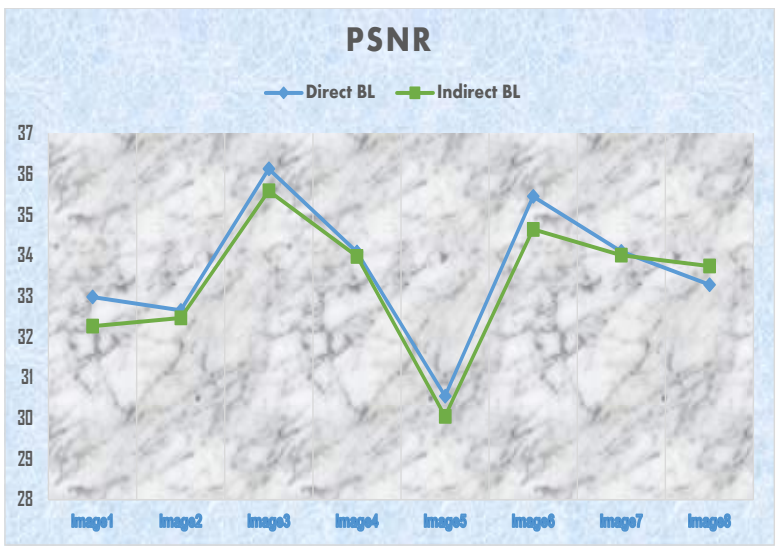

Figure 8: BL-based results for direct and indirect models in medical images.

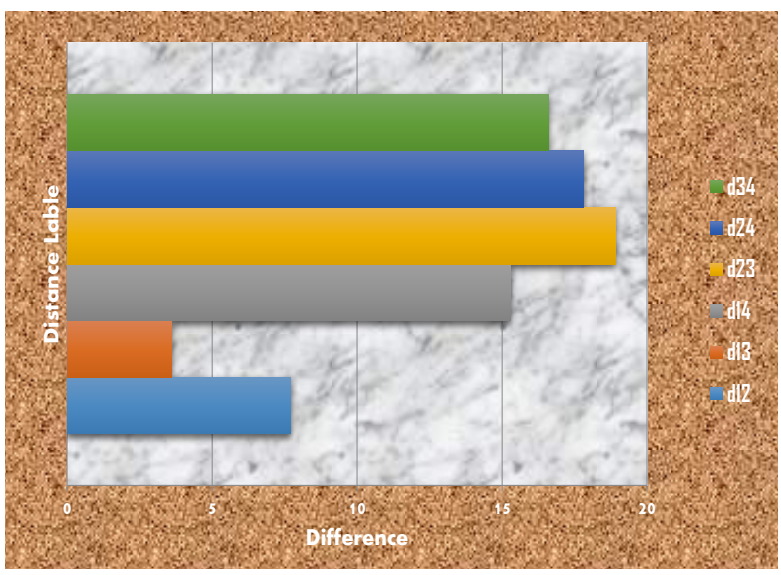

Figure 9: It shows the distances of all models compared each other. 
Table 4: Details for Figure 9

\begin{tabular}{|c|c|c|}
\hline Distance & $\begin{array}{l}\text { Interpolation } \\
\text { Methods }\end{array}$ & Description \\
\hline $\mathrm{d} 12$ & $\begin{array}{l}\text { Direct CC, } \\
\text { Indirect CC }\end{array}$ & Difference in Fig. 7 \\
\hline d13 & $\begin{array}{l}\text { Direct BL, } \\
\text { Indirect BL }\end{array}$ & $\begin{array}{c}\text { Minimum difference, } \\
\text { Difference in Fig. } 8\end{array}$ \\
\hline d14 & $\begin{array}{l}\text { Direct } \mathrm{BL} \text {, } \\
\text { Indirect CC }\end{array}$ & - \\
\hline$d 23$ & $\begin{array}{l}\text { Direct CC, } \\
\text { Indirect BL }\end{array}$ & Maximum difference \\
\hline $\mathrm{d} 24$ & $\begin{array}{l}\text { Direct CC, } \\
\text { Direct BL }\end{array}$ & - \\
\hline d34 & $\begin{array}{l}\text { Indirect BL, } \\
\text { Indirect CC }\end{array}$ & - \\
\hline
\end{tabular}

that selecting original pixels might not have a considerable impact on the algorithm performance. In other words, some interpolators in medical dataset can be affected by the selection models, while, some cannot. In addition, computational complexity [15] for direct and indirect models should be further discussed in terms of number of operations. For a future work, we can consider these results as a guideline for improving non-freesize interpolators to a more free-size state or generally other interpolators [16-22] and image processing applications such as [9, 23-24].

\section{Conclusion}

The analyses of the outputs show that ignoring the manner of reconstruction models is clearly effective in numerical outputs. The
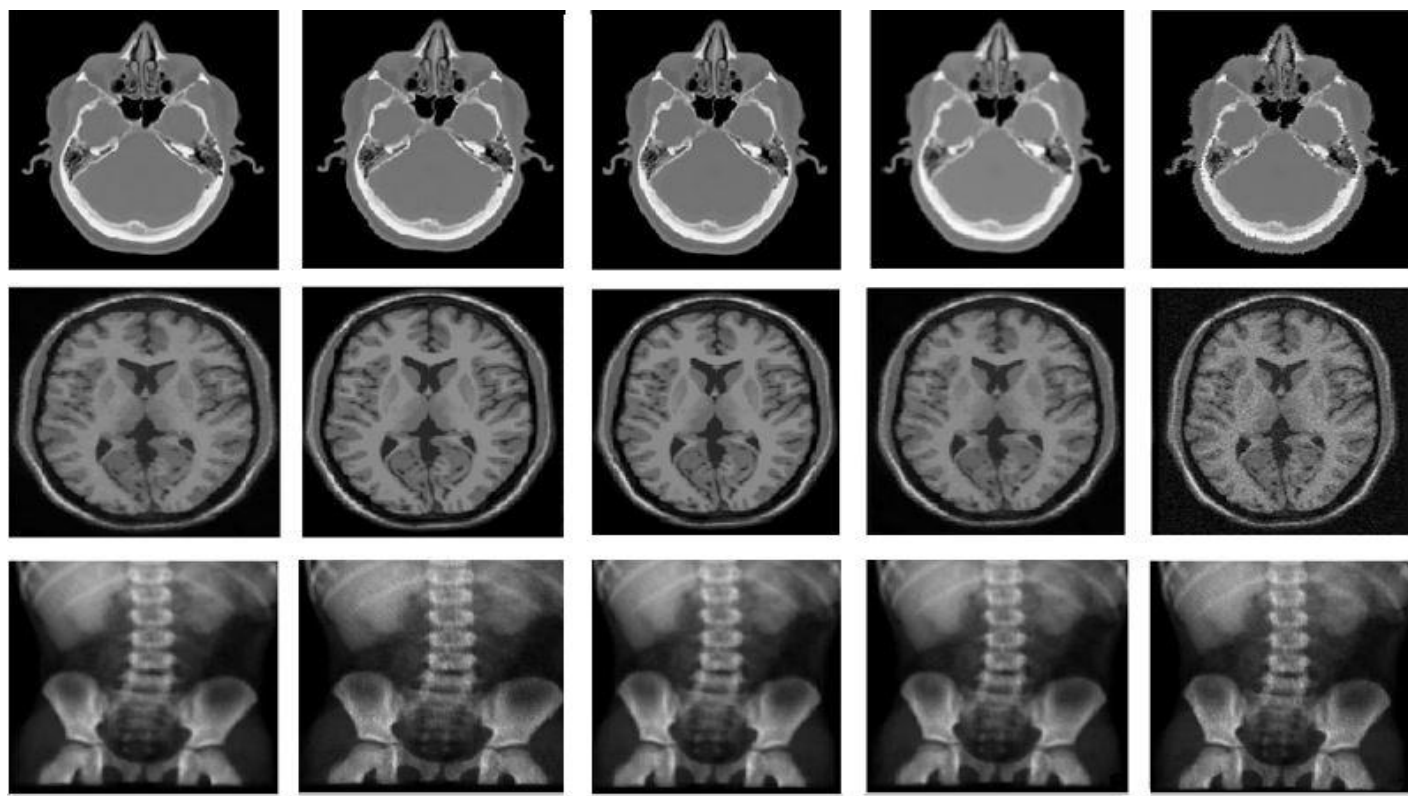

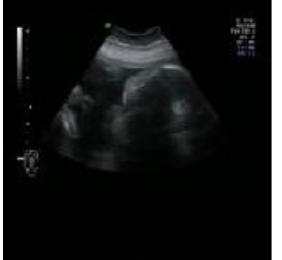

A

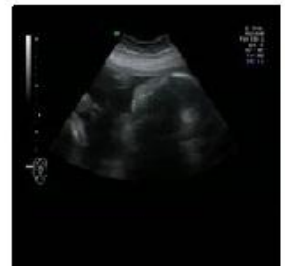

B

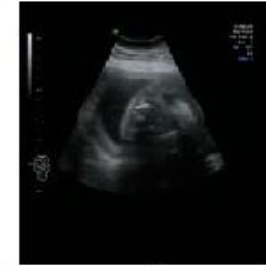

C

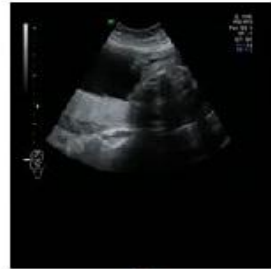

D

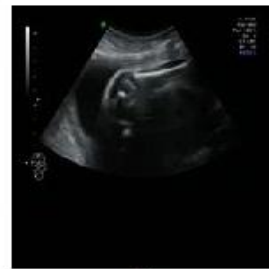

E

Figure 10: Columns " $B$ ", " $C$ ", " $D$ ", and " $E$ " show the different models of Direct BL, Direct CC, Indirect $B L$, and Indirect $C C$, respectively; and the column " $A$ " shows the source images before any processing including down-sampling and reconstruction. Direct $\mathrm{CC}$ model has the best quality in practice. 
Medical Image Magnification

current research exhibited that selection models are not a general factor in reconstruction problems, and the structure of the basic interpolators is also a main factor which affects the final results. Therefore, in designing new magnification algorithms for direct zoom of medical images, it is possible that selecting original pixels will not have a considerable impact on the algorithm performance. In other words, some interpolators in medical dataset can be affected by selection models, but some cannot.

\section{Acknowledgment}

Thanks to the Editor-in-Chief and his colleagues in the journal editorial office for their kind support.

\section{Conflict of Interest}

There is no conflict of interest.

\section{References}

1. Gonzalez RC, Woods RE. Digital image processing. Upper Saddle River, NJ: Prentice Hall; 2012.

2. Khosravi M, Rostami H. Improving the Binary Watermark-based Data Hiding Scheme in Remote Sensing Images. ICAUCAE; Tehran: SID; 2016.

3. Khosravi MR, Sharif-Yazd M, Moghimi MK, Keshavarz A, Rostami H, Mansouri S. MRF-based multispectral image fusion using an adaptive approach based on edge-guided interpolation. Journal of Geographic Information System. 2017; 9:114-25.

4. Khosravi M, Keshavarz A, Rostami H, Mansouri S, editors. Statistical Image Fusion for HR Band Colorization in Landsat Sensors. 20th Annual Conference of Computer Society of Iran (CSICC2015); Mashhad: CSI; 2015. p. 245-50.

5. Unser M, Thevenaz P, Yaroslavsky L. Convolution-based interpolation for fast, high-quality rotation of images. IEEE Trans Image Process. 1995:4:1371-81. doi: 10.1109/83.465102. PubMed PMID: 18291969.

6. Maeland E. On the comparison of interpolation methods. IEEE Trans Med Imaging. 1988;7:2137. doi: 10.1109/42.7784. PubMed PMID: 18230471.

7. Alhihi M. Network Coding for Wireless Sensor Network Cluster over Rayleigh Fading Channel:
Finite State Markov Chain. International Journal of Communications, Network and System Sciences. 2017;10:1.

8. Alhihi M. Practical routing protocol models to improve network performance and adequacy. Journal of Computer and Communications. 2017;5:114-24.

9. Moghimi MK, Pourghassem H. Shadow Detection Based on Combinations of Hessenberg Decomposition and Principal Component Analysis in Surveillance Applications. IETE Journal of Research. 2015;61:269-84.

10. Keys R. Cubic convolution interpolation for digital image processing. IEEE Trans Acoust. 1981;29:1153-60.

11. Hou $H$, Andrews $H$. Cubic splines for image interpolation and digital filtering. IEEE Trans Acoust. 1978;26:508-17.

12. Parker JA, Kenyon RV, Troxel DE. Comparison of interpolating methods for image resampling. IEEE Trans Med Imaging. 1983;2:31-9.

13. Khosravi M, Rostami H. A New Statistical Technique for Interpolation of Landsat Images. ICAUCAE 2016; Tehran: SID; 2016.

14. Khosravi MR. Single- and Multi-Step Image Enlargement for Medical Image Coding. Current Signal Transduction Therapy. 2019;14:1-2.

15. Khosravi MR, Basri $H$, Khosravi A, Rostami $H$, editors. Energy efficient spherical divisions for VBF-based routing in dense UWSNs. 2nd International Conference on Knowledge-Based Engineering and Innovation (KBEI); Tehran: IEEE; 2015. p. $961-5$

16. Unser M, Aldroubi A, Eden M. Fast B-spline transforms for continuous image representation and interpolation. IEEE Transactions on pattern analysis and machine intelligence. 1991;13:27785.

17.Dodgson NA. Quadratic interpolation for image resampling. IEEE Trans Image Process. 1997;6:1322-6. doi: 10.1109/83.623195. PubMed PMID: 18283021.

18. Schultz RR, Stevenson RL. A Bayesian approach to image expansion for improved definition. IEEE Transactions on Image Processing. 1994;3:23342.

19. Thurnhofer S, Mitra SK. Edge-enhanced image zooming. Optical Engineering. 1996;35:1862-71.

20. Grevera GJ, Udupa JK, Miki Y. A task-specific evaluation of three-dimensional image interpolation techniques. IEEE Trans Med Imag- 
Akbarzadeh O. et al

ing. 1999;18:137-43. doi: 10.1109/42.759116.

PubMed PMID: 10232670.

21. Appledorn CR. A new approach to the interpolation of sampled data. IEEE Trans Med Imaging. 1996;15:369-76. doi: 10.1109/42.500145. PubMed PMID: 18215917.

22. Lee $C$, Eden $M$, Unser M. High-quality image resizing using oblique projection operators. IEEE Trans Image Process. 1998;7:679-92. doi: 10.1109/83.668025. PubMed PMID: 18276284.

23. Khosravi MR, Khosravi A, Shadloo-Jahromi M,
Keshavarz A, editors. A novel fake color scheme based on depth protection for MR passive/optical sensors. 2nd International Conference on Knowledge-Based Engineering and Innovation (KBEI); Tehran: EEE; 2015. p. 362-7.

24. Khosravi MR, Rostami H, Samadi S. Enhancing the Binary Watermark-Based Data Hiding Scheme Using an Interpolation-Based Approach for Optical Remote Sensing Images. International Journal of Agricultural and Environmental Information Systems (IJAEIS). 2018;9:53-71. 